\title{
Residue Classes of the Partition Function
}

\author{
By D. W. MacLean
}

Abstract. The results of computations of $p(n)\left(\bmod p^{k}\right)$ for primes $p<100$ are summarized in frequency tables of residue classes.

1. Introduction. If $p(n)$ denotes the number of unrestricted partitions of $n$, let

$$
\begin{aligned}
& A(m, k, x)=\{n: p(n) \equiv k(\bmod m) \text { and } n \leqslant x\}, \\
& A(m, k)=\{n: p(n) \equiv k(\bmod m)\}, \\
& s(m, k, x)=\operatorname{card} A(m, k, x), \text { and let } \\
& d(m, k, x)=s(m, k, x) / x .
\end{aligned}
$$

In 1960, Morris Newman [9] conjectured that $p(n)$ fills all residue classes $\bmod m$ infinitely often. In our notation, this is equivalent to the conjecutre that no $A(m, k)$ is finite.

Proofs of this conjecture have been discovered for $m=2$ ([6]), 5 ([9]), 7 ([1]), 13 ([9]), 17, 19, 29, 31 ([4]), and $11^{2}$ ([5]). The Ramanujan congruences imply that $A\left(5^{a} 7^{b} 11^{c}, 0\right)$ is infinite for all natural numbers $a, b$, and $c$.

It is natural to ask the following questions about the densities and structures of the $A(m, k)$ 's:

1. Does $\lim _{x \rightarrow \infty} d(m, k, x)$ exist?

2. Is $\lim _{x \rightarrow \infty}$ inf $d(m, k, x)>0$ ?

3. Does $A(m, k)$ contain any arithmetic progressions or any other simple infinite sequences?

2. Tables of Residue Classes. Using a PDP $11 / 60, A(m, k, 19000)$ was computed for $m$ equal to powers of $2,3,5,7$, and 11 less than $5^{5}$, and for $m$ equal to primes less than 100. A summary of the results is contained in the following tables.

TABLE 1. Values of $s\left(5^{\alpha}, 0,19000\right)$

\begin{tabular}{|c|c|c|c|c|c|c|}
\hline $\mathrm{m}$ & 5 & $5^{2}$ & $5^{3}$ & $5^{4}$ & $5^{5}$ & $5^{6}$ \\
\hline $\mathrm{s}(\mathrm{m}, 0,19000)$ & 6907 & 1992 & 724 & 172 & 57 & 5 \\
\hline
\end{tabular}

Received November 20, 1978.

AMS (MOS) subject classifications (1970). Primary $10 \mathrm{~A} 45$. 
TABLE 2. Values of $s\left(7^{\alpha}, 0,19000\right)$

\begin{tabular}{|c|c|c|c|c|}
\hline$m$ & 7 & $7^{2}$ & $7^{3}$ & $7^{4}$ \\
\hline$s(m, 0,19000)$ & 5244 & 2070 & 308 & 47 \\
\hline
\end{tabular}

TABLE 3. Values of $s\left(5^{2}, 5 l+j, 19000\right)$

\begin{tabular}{|c|c|c|c|c|c|}
\hline$l$ & 0 & 1 & 2 & 3 & 4 \\
\hline 0 & 1992 & 587 & 615 & 595 & 596 \\
\hline 1 & 1240 & 578 & 631 & 609 & 575 \\
\hline 2 & 1249 & 587 & 655 & 617 & 649 \\
\hline 3 & 1220 & 567 & 663 & 573 & 610 \\
\hline 4 & 1206 & 559 & 604 & 594 & 630 \\
\hline
\end{tabular}

TABLE 4. Values of $s\left(7^{2}, 7 l+j, 19000\right)$

\begin{tabular}{|c|c|c|c|c|c|c|c|}
\hline$\ell$ & 0 & 1 & 2 & 3 & 4 & 5 & 6 \\
\hline 0 & 2070 & 308 & 318 & 363 & 362 & 317 & 332 \\
\hline 1 & 512 & 298 & 304 & 323 & 327 & 339 & 328 \\
\hline 2 & 495 & 332 & 329 & 317 & 327 & 327 & 302 \\
\hline 3 & 508 & 376 & 340 & 318 & 336 & 328 & 355 \\
\hline 4 & 526 & 303 & 344 & 326 & 308 & 307 & 333 \\
\hline 5 & 526 & 332 & 313 & 333 & 342 & 300 & 341 \\
\hline 6 & 559 & 342 & 350 & 363 & 338 & 310 & 314 \\
\hline
\end{tabular}


TABLE 5. Values of $s\left(11^{2}, 11 l+j, 19000\right)$

\begin{tabular}{|c|c|c|c|c|c|c|c|c|c|c|c|}
\hline$\ell$ & 0 & 1 & 2 & 3 & 4 & 5 & 6 & 7 & 8 & 9 & 10 \\
\hline 0 & 487 & 138 & 164 & 139 & 151 & 160 & 159 & 138 & 134 & 148 & 163 \\
\hline 1 & 290 & 160 & 143 & 131 & 147 & 141 & 138 & 135 & 148 & 148 & 138 \\
\hline 2 & 309 & 200 & 145 & 127 & 150 & 133 & 144 & 125 & 154 & 128 & 143 \\
\hline 3 & 272 & 158 & 130 & 116 & 143 & 150 & 138 & 146 & 154 & 146 & 145 \\
\hline 4 & 276 & 132 & 154 & 124 & 123 & 154 & 137 & 124 & 143 & 161 & 123 \\
\hline 5 & 281 & 132 & 143 & 138 & 162 & 128 & 126 & 146 & 140 & 129 & 141 \\
\hline 6 & 295 & 117 & 138 & 126 & 144 & 155 & 136 & 139 & 136 & 146 & 138 \\
\hline 7 & 269 & 143 & 131 & 126 & 154 & 118 & 147 & 146 & 134 & 151 & 131 \\
\hline 8 & 317 & 152 & 157 & 163 & 136 & 141 & 148 & 162 & 145 & 134 & 137 \\
\hline 9 & 307 & 128 & 122 & 147 & 153 & 143 & 140 & 149 & 167 & 149 & 118 \\
\hline 10 & 279 & 130 & 139 & 126 & 136 & 137 & 156 & 128 & 154 & 164 & 144 \\
\hline
\end{tabular}

Tables of $s(m, k, 19000)$ for $m$ running through the primes from 29 to 97 , as well as listings of $A(m, 0,19000)$ for $m=5^{2}, 5^{3}, 5^{4}, 5^{5}, 7^{2}, 7^{3}, 7^{4}, 11^{2}, 11^{3}, 11^{4}$, have been deposited in the UMT file.

3. Calculation of $p(n)$. A table of values of $p(n), n \leqslant 1435$, has been deposited in the UMT file. For $n \leqslant 600$, the values obtained agree with those of Gupta [3]. The value $p(721)=161061755750279477635534762$ is equal to that obtained by D. H. Lehmer [7]. As an additional check, it was determined that $13 \mid p(n)$ for $n=747,890,903,968,1176$, and 1241 , as predicted by M. Newman [8].

4. Conclusion. It is hoped that examination of the tables will lead to explanations of the observed values of the residue classes of $p(n)$ and to answers of the questions posed in Section 1. Kl $\phi$ ve [5] has shown that

$$
\begin{aligned}
& \lim \inf d(5,0, x) \geqslant .20194, \\
& \lim \inf d(7,0, x) \geqslant .150697143, \\
& \lim \inf d(13,0, x) \geqslant .00204,
\end{aligned}
$$

while our calculations give $d(5,0,19000)=.363507184, d(7,0,19000)=$ $.273459292, d(13,0,19000)=.079416873$, showing that there may be considerable room for improvement of the theory. 


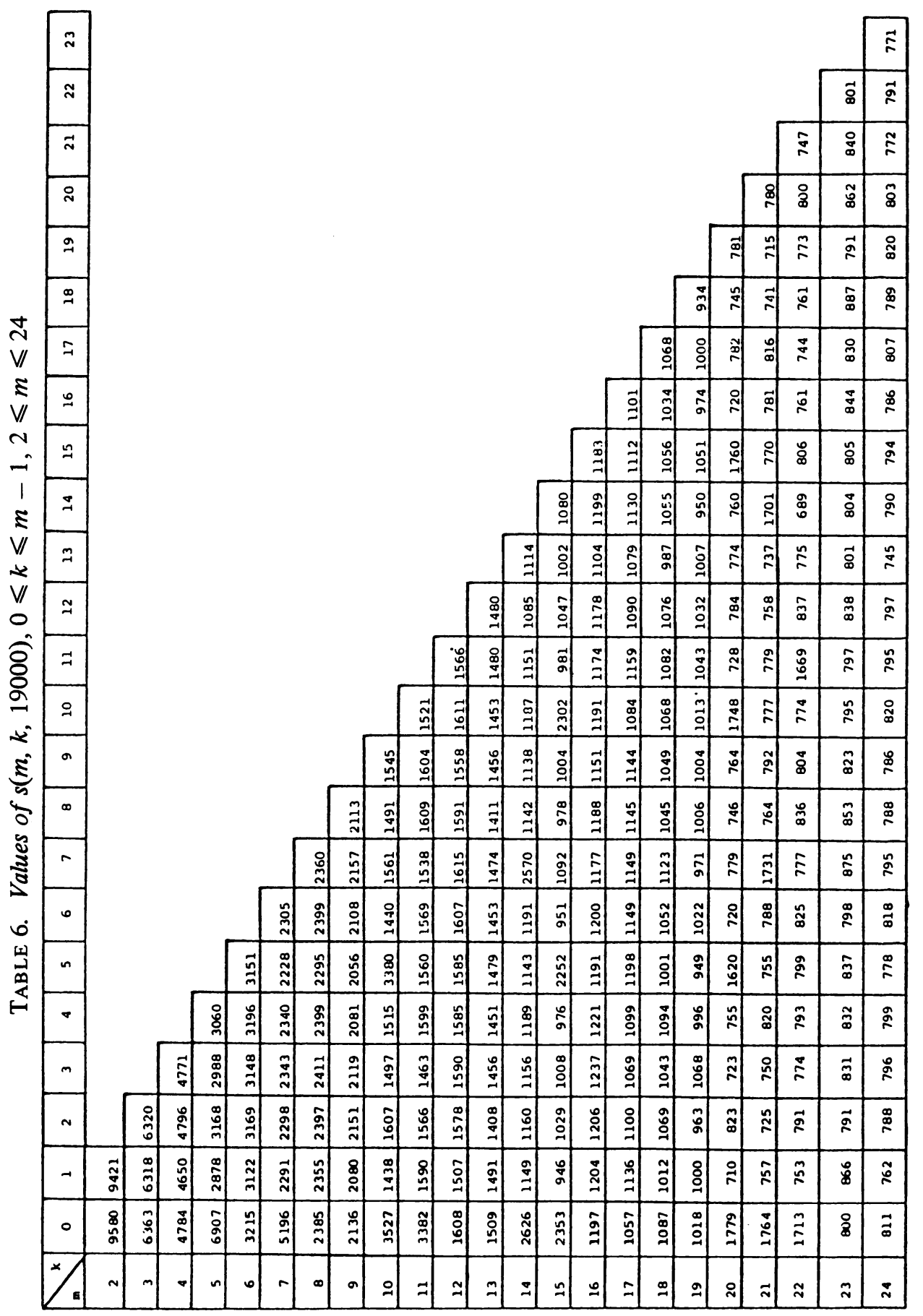


Department of Mathematics

University of Saskatchewan

Saskatoon, Canada S7N oWo

1. A. O. L. ATKIN, "Multiplicative congruence properties and density problems for $p(n)$," Proc. London Math. Soc. (3), v. 18, 1968, pp. 563-567.

2. A. O. L. ATKIN \& J. N. O'BRIEN, "Some properties of $p(n)$ and $c(n)$ modulo powers of 13," Trans. Amer. Math. Soc., v. 126, 1967, pp. 442-459.

3. H. GUPTA, A. E. GWYTHER \& J. C. P. MILLER, "Tables of partitions," Roy. Soc. Math. Tables, v. 4, 1958.

4. TORLEIV KL $\emptyset \mathrm{VE}$, "Recurrence formulae for the coefficients of modular forms and congruences for the partition function and for the coefficients of $j(\tau),(j(\tau)-1728)^{1 / 2}$ and $j(\tau)^{1 / 3}$," Math. Scand., v. 23, 1968, pp. 133-159.

5. TORLEIV KL $\emptyset$ VE, “Density problems for $p(n)$," J. London Math. Soc. (2), v. 2, 1970, pp. 504-508.

6. O. KOLBERG, "Note on the parity of the partition function," Math. Scand., v. 7, 1959, pp. $377-378$.

7. D. H. LEHMER, "On the series for the partition function," Trans. Amer. Math. Soc., v. 43, 1938, pp. $271-295$.

8. MORRIS NEWMAN, "Congruences for the coefficients of modular forms and some new congruences for the partition function," Canad. J. Math., v. 9, 1957, pp. 549-552.

9. MORRIS NEWMAN, "Periodicity modulo $m$ and the divisibility properties of the partition function," Trans. Amer. Math. Soc., v. 97, 1960, pp. 225-236.

10. MORRIS NEWMAN, "Note on partitions modulo 5," Math. Comp., v. 21, 1967, pp. $481-482$.

11. THOMAS R. PARKIN \& DANIEL SHANKS, "On the distribution of parity in the partition function," Math. Comp., v. 21, 1967, pp. 466-480.

12. S. RAMANUJAN, "Congruence properties of partitions," Proc. London Math. Soc. (2), v. 18,1920 .

13. G. N. WATSON, “Ramanujan's Vermutung über Zerfällungsanzahlen,” J. Reine Angew. Math., v. 179, 1938, pp. 97-128. 